\title{
Electric Energy Substitution Potential Prediction Based on Logistic Curve Fitting and Improved BP Neural Network Algorithm
}

\author{
Yujun Liu, Cheng Hu, Yi Hong \\ School of Electronical Engineering, Southeast University, \\ 210096 Nanjing, China \\ liuyujun@seu.edu.cn
}

\begin{abstract}
In order to predict the potential of electric energy substitution in the next decade in China, this paper proposes a prediction method based on Logistic curve fitting and improved BP neural network algorithm. The amount of electric energy substitution is defined to quantify the potential of electric energy substitution. Then the important influencing factors of electric energy substitution based on the Impact by Population, Affluence and Technology (IPAT) model are established and quantified. For different influencing factors, logistic curve fitting and polynomial function fitting method were used to estimate the data fitting. A two-node output layer model of BP neural network is established and improved with additional momentum factors and adaptive learning rate to learn and train the data related to electric energy substitution from 2003 to 2017, and calculate the amount of electric energy substitution which are substitution potential from 2018 to 2020,2025 and 2030. The calculation results show that the method has higher computational accuracy and fewer iterations. The prediction results are reasonable and effective, which can be the reference of the research of energy substitution.
\end{abstract}

Index Terms-Electric energy substitution; Potential prediction; BP neural network algorithm; Logistic curve fitting.

\section{INTRODUCTION}

With the development of productivity, human consumption and dependence on fossil energy is increasing. As a non-renewable resource, fossil energy is facing the risk of shortage and depletion [1]. Electric energy, a clean and convenient secondary energy source which can be used in high efficiency, is increasingly favoured by the state and the government. The substitution of electric energy refers to substitute the consumption of fossil energy such as coal, oil and natural gas in energy consumption, and the increase of the proportion of electric energy in the terminal energy consumption [2]. The development and consumption of renewable energy resources can be promoted, and the supply cost of electricity can be reduced by carrying out electric energy substitution widely which can promote the upgrading of energy-related industries, stimulate the development of the global economy, and ultimately achieve the common development of humanity around the world [3].

Manuscript received 28 September, 2018; accepted 14 February, 2019. This research was funded by National key research and development projects of China (No. 2017YFA0700300).
The National Development and Reform Commission and the Energy Bureau of China proposed clearly to increase the substitution of electric energy to save energy and promote green development, and put forward the goal of replacing 450 billion kWh of electric energy in the "13th Five-Year Plan for Power Development" [4]. Therefore, it is valuable to research the calculation of the substitution amount of electric energy and the prediction of the substitution potential.

In view of the prediction of electric energy substitution potential, domestic scholars have proposed many research methods. In reference [5], a research model of electric energy substitution potential based on Stochastic Impacts by Regression on Population, Affluence and Technology (STIRPAT) is proposed to quantitatively analyse the relationship between the potential of electric energy substitution and its influencing factors. Reference [6] refers to the IPAT equation construction method and establishes an IPAT model for electric energy replacement to evaluate the potential of electric energy replacement. A variety of electric energy substitution scenarios are designed to nest the multivariate nonlinear regression combined with the wavelet neural network intelligent correction prediction model in the IPAT model. Literature [7] proposed a method of energy substitution potential analysis based on particle swarm optimization support vector machine (PSO-SVM), and verified its validity by historical data from 2010 to 2014 in China. Literature [8] explored the regional electric energy substitution potential, and used the regional Gross Domestic Product (GDP), population, coal and other energy consumption as the potential evaluation indicators, and established a regional multi-scenario electric energy substitution potential analysis model and the energy consumption through grey model (GM) prediction model. Forecasting is carried out to predict the substitution potential of electric energy in various scenarios.

Learning from the above methods, this paper combines Logistic curve fitting and back propagation (BP) network learning algorithm to predict the potential of electric energy substitution. The IPAT model is improved and the quantitative factors affecting the Electric energy substitution by Population, Affluence, Technology and Regulation (EPATR) model are established. The logistic curve or polynomial function is used to fit the estimate for different 
influencing factors. The improved BP neural network model is established. The direct substitution of electricity and indirect substitution of electricity is used as the output nodes. The additional momentum factor and adaptive learning rate are introduced. The learning and test data are randomly assigned, and the predicted values export after the iteration reaches the set precision. The example shows that the improved BP algorithm has higher computational accuracy and shorter iteration time. The prediction results are compared with the time series prediction method and the least squares prediction method to verify its rationality.

\section{Substitution Potential Model}

\section{A. Substitution Styles}

Electric energy substitution involves many fields such as residential heating, industrial and agricultural production, transportation, power supply and consumption [9]. According to the specific field and alternative forms of application, it can be divided into direct substitution style and indirect substitution style, as shown in Fig. 1.

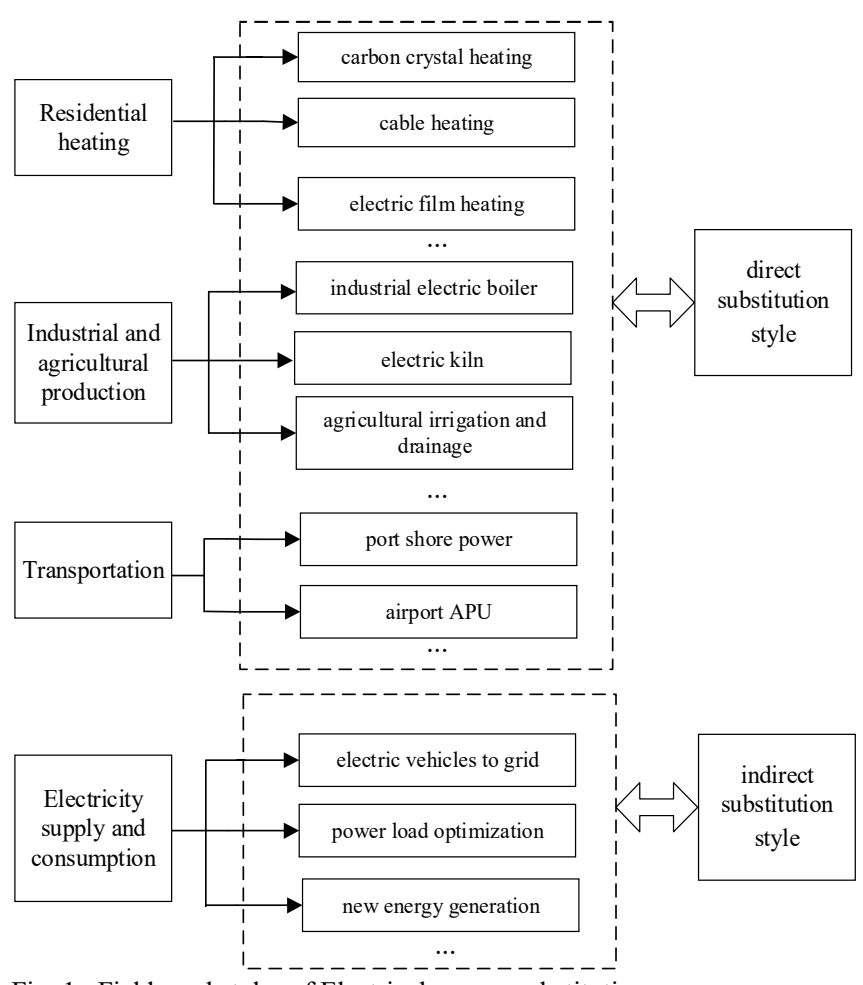

Fig. 1. Fields and styles of Electrical energy substitution.

Direct substitution style is defined as the substitution of fossil energy that can be used with electrical energy directly. The indirect substitution style is defined as: reducing the amount of fossil energy power generation by means of energy storage, peak clipping and valley filling, and promotion of new energy consumption to improve the utilization efficiency of electric energy.

\section{B. Amount of Electric Energy Substitution}

The amount of electric energy substitution is used as the basis for electric energy substitution potential. It can be computed as follows

$$
E_{s u b}(t)=E_{d}(t)+E_{\text {in }}(t),
$$

where $E_{s u b}(t)$ is the amount of electric energy substitution, $E_{d}(t)$ is the amount of direct substitution, $E_{\text {in }}(t)$ is the amount of indirect substitution, $t$ is the year.

The amount of direct substitution can be calculated by thermal equivalent method [10]

$$
E_{d}(t)=\sum_{i=1}^{n} M_{i}(t) C_{i} h_{i} /(3600 \times 95 \%),
$$

where $i=1,2,3 \ldots, M_{i}(t)$ is the amount of alternative fossil energy, $C_{i}$ is combustion value of fossil energy, $h_{i}$ is thermal benefit value of fossil energy, $i$ is the number of the kind of fossil energy.

The amount of indirect substitution can be calculated by data of power system and new energy generation [11]. Considering that the electric energy substitution amount is the cumulative value, it is necessary to observe the electric energy substitution growth within one year in the actual prediction calculation, which is the electric energy substitution amount increment

$$
\Delta E_{s u b}(t)=E_{s u b}(t)-E_{s u b}(t-1)
$$

where $\Delta E_{\text {sub }}(t)$ is the electric energy substitution amount increment.

\section{Influencing Factors of Substitution Amount}

The potential of electric energy substitution amount is the result of a combination of various factors. This paper refers to the IPAT model of environmental load equation to establish the IPAT model of electric energy substitution potential [12]. The potential of electric energy substitution amount is closely related to population $(P)$, wealth $(A)$, technology development $(T)$, and policy support $(R)$. These four factors are used as the most important factors affecting electric energy substitution to establish EPATR model

$$
\begin{gathered}
E_{s u b}(t)=E_{s u b}(t-1)+ \\
+\Delta E_{s u b}(t-1)\left[k_{1} \frac{A(t-1)}{P(t-1)} \times k_{2} T(t-1) \times k_{3} R(t-1)\right],
\end{gathered}
$$

where $k_{1}$ is richness elasticity coefficient, $k_{2}$ is technical level elastic coefficient, $k_{3}$ is policy support elasticity coefficient.

According to (4), the increase in electric energy substitution is closely related to the previous year's population, wealth, technology development, and policy support. The four important influencing factors must be quantified first.

1. Population quantification $P(t)$ :

In this paper, population is used as a parameter to influence the amount of substitution.

2. Wealth quantification $A(t)$ :

Per capita GDP is used as the quantification of wealth.

3. Technology development quantification $T(t)$ :

It is defined as follows

$$
T(t)=\frac{E(t)}{E(t)+E_{f}(t)} \times 100 \%,
$$

where $E(t)$ is the electricity consumption amount, $E_{f}(t)$ is 
fossil energy consumption equivalent power, its calculation can refer to (2).

4. Policy support quantification $R(t)$ :

It is defined as follows

$$
R(t)=\frac{\lambda_{e}(t)}{\lambda_{e}(t)+\lambda_{c o}(t)+\lambda_{p e}(t)+\lambda_{g a}(t)} \times 100 \%,
$$

where $\lambda_{e}(t)$ is Chinese investment in the amount of power assets, $\lambda_{c o}(t)$ is China's coal mining investment assets, $\lambda_{p e}(t)$ is oil exploitation and processing investment assets, $\lambda_{g a}(t)$ is natural gas extraction and processing investment assets.

\section{PREDICTION METHOD}

This section combines the actual situation and development trend of each influencing factor, and uses Logistic curve to estimate it. The estimated value is used as the input value of the trained BP neural network to obtain the predicted electric energy substitution amount, that is, the electric energy substitution potential.

\section{A. Logistic Curve Fitting}

The Logistic curve is a common sigmoid function, named after Pierre François Velul in 1844 or 1845 when studying its relationship with population growth. The Logistic function expression is

$$
y(x)=\frac{K P_{0} \exp [r x]}{K+P_{0}(\exp [r x]-1)},
$$

where $P_{0}$ is the initial value, $K$ is the end value, $r$ is the value of the curve change. The function image is shown as follows when $K=1, P_{0}=0.5, r=1$.

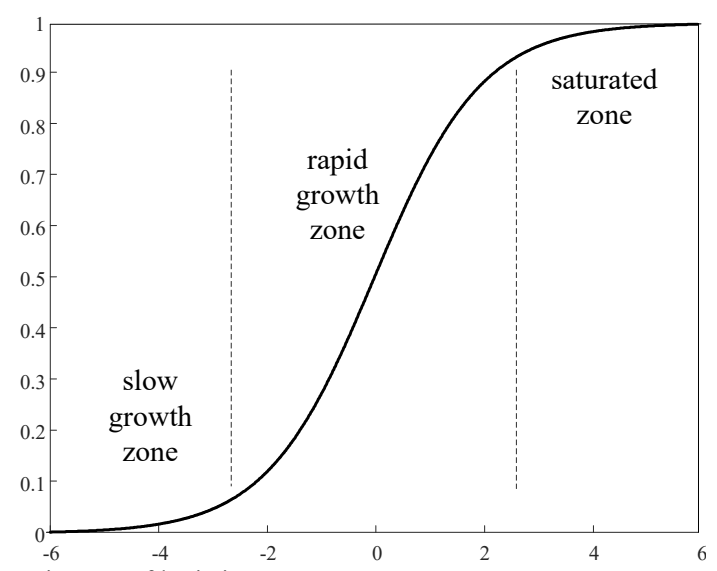

Fig. 2. Diagram of logistic curve.

It can be seen from Fig. 2 that the Logistic curve is roughly divided into three regions: a slow growth zone, a growth fast zone and a saturation zone. China's population is experiencing aging. In 2017, the birth rate was only $12.43 \%$, and the natural population growth rate dropped to a low fertility level of $5.32 \%$. The future population growth rate is low [13]; China's energy consumption has gradually eased in recent years; Therefore, population, fossil energy consumption growth can be fitted with a saturation zone curve. China's economic level has entered a new normal period of development, and the growth rate of GDP growth in the future is stable at around $6.7 \%$. The same level of electric energy substitution technology is in a period of vigorous development; therefore, GDP and technical level growth can be fitted by the growth fast-zone curve.

\section{B. Improved BP Neural Network Algorithm}

The BP (Back Propagation) neural network is a multi-layer feedforward network algorithm trained by the error back propagation algorithm proposed by the group of scientists led by Rumelhart and McCelland in 1986 [14]. The basic idea is to continuously correct the weight and threshold of the system according to the error propagation of the output value and the target value until the iteration accuracy is satisfied.

The internal calculation process of BP network and the process of predicting electric energy substitution amount are shown in Fig. 3.

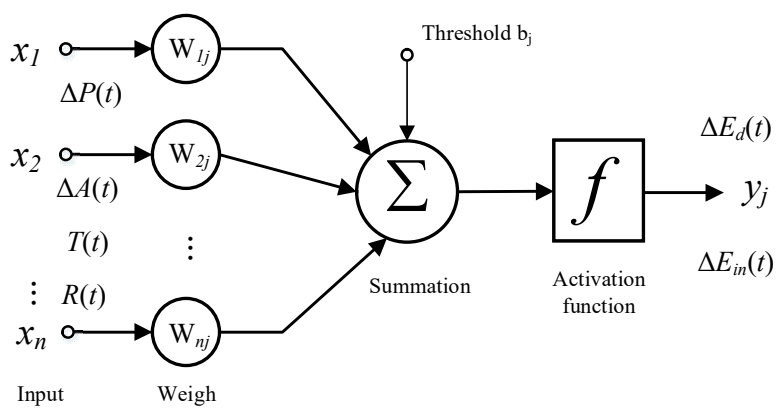

Fig. 3. Diagram of predicting the amount of energy replacement with BP neural network.

Since BP neural network is easy to fall into the local minimum of the error surface [15], and different learning rates affect the stability and training time of the system [16], this paper improves the BP network according to the two defects, mainly introduces the additional Momentum factor and adaptive learning rate.

The weight adjustment formula with additional momentum factor is as follows

$$
\Delta w_{i j}(S)=-\eta \times \frac{\partial \varepsilon_{j}}{\partial w_{i j}}+\alpha \Delta w_{i j}(S-1)
$$

where $i=1,2 \ldots n, j=1,2 \ldots m, S=1,2 \ldots, \Delta w_{i j}(S)$ is the weight deviation, $\varepsilon_{j}$ is the overall error, $w_{i j}$ is the input weight, $\eta$ is the learning rate, $\alpha$ is weight coefficient correction constant, $S$ is the number of iterations, $n$ is the amount of input variables, $m$ is the amount of output variables.

The adaptive learning rate adjustment settings are as follows

$$
\eta(S+1)=\left\{\begin{array}{cc}
1.05 \eta(S), & \varepsilon(S+1)<\varepsilon(S) \\
0.7 \eta(S), & \varepsilon(S+1)>1.04 \varepsilon(S), \\
\eta(S), & \text { else. }
\end{array}\right.
$$

\section{Improved BP Algorithm Steps}

Step 1) Design network structure and initialization

Design the number of the network output layer, hidden layer and output layer nodes, set the error function e, set the calculation precision I, set the initial learning rate, adjust the adaptive learning rate with reference to (9), and set the 
maximum learning frequency $M$.

Step 2) Computing network output

$$
y_{j}(k)=f\left(\sum_{i=1}^{n}\left(w_{i j} x_{i j}(k)\right)+b_{u j}\right),
$$

where $i, k=1,2 \ldots n, j=1,2 \ldots m, u=1,2 \ldots q, y_{j}(k)$ is the output value, $x_{i j}(k)$ is the input value, $b_{u j}$ is the threshold value, and $i$ is the input number, $u$ is the hide node number, $j$ is the output number, $k$ is the sample number, $q$ is the amount of hidden layer variables.

Step 3) Computing the overall error

$$
\varepsilon_{j}=\sum_{k=1}^{n} \frac{1}{2}\left(d_{j}(k)-y_{j}(k)\right)^{2}
$$

where $k=1,2 \ldots n, j=1,2 \ldots m, d_{j}(k)$ is the expected output value.

Step 4) Reverse partial derivative correction

$$
\frac{\partial \varepsilon_{j}}{\partial w_{i j}}=\frac{\partial \varepsilon_{j}}{\partial f} \times \frac{\partial f}{\partial\left(\sum_{i=1}^{n}\left(w_{i j} x_{i j}(k)\right)\right)} \times \frac{\partial\left(\sum_{i=1}^{n}\left(w_{i j} x_{i j}(k)\right)\right)}{\partial w_{i j}}
$$

where $k=1,2 \ldots n j=1,2 \ldots m$. The update of the weight refers to (8), and a new round of weights is input into the network to calculate an update threshold.

Step 5) Repeat iteration to set accuracy

After updating the weight and the threshold, the calculation is started from the above step 2 until the calculation error is less than the set calculation precision $I$. At this time, it is a network completed by training, and the corresponding output value can be obtained according to a certain estimated input value to realize the prediction function.

\section{Substitution Potential Prediction Process}

The electric energy substitution potential prediction process is shown in Fig. 4. It is worth noting that this paper uses the form of annual increments as the input and output of the network, including population, GDP, direct substitution amount and indirect substitution amount. Due to the demographic factor, the factor of wealth has a great influence on the direct substitution of electricity, and the development of technology has a greater impact on the indirect substitution of electricity. Different influencing factors have different emphasis on the two styles. So the output layer adopts two nodes, which are direct substitution amount and indirect substitution amount. Then the total substitution amount will be acquired.

\section{CASE ANALYSIS}

This case predicts the amount of energy substitution between 2018 and 2020 and 2025 and 2030 based on data from China's electric energy substitution from 2003 to 2017. The raw data for the substitution of electricity from 2003 to 2017 is shown in Table I.

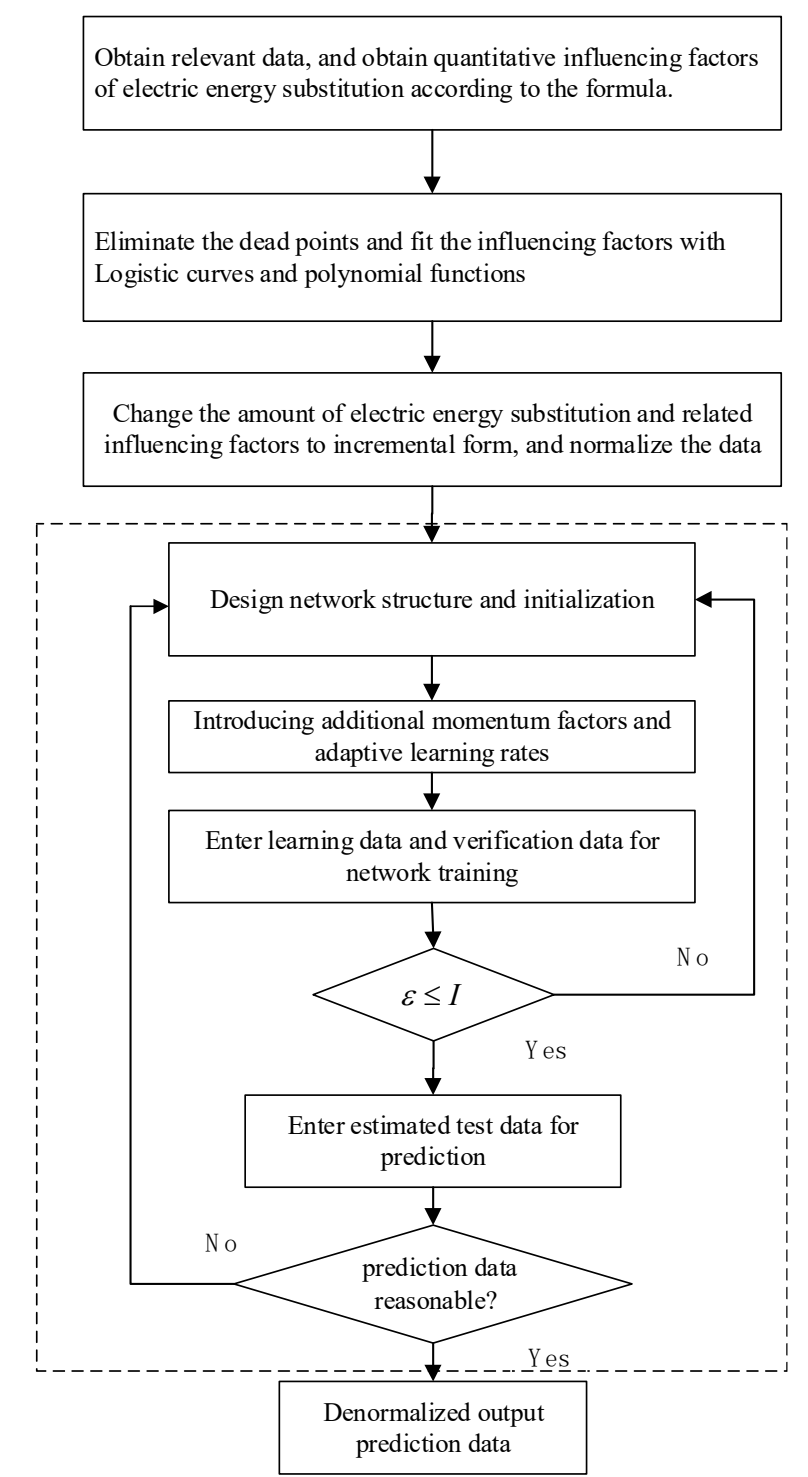

Fig. 4. Diagram of predicting the amount of energy substitution with

\begin{tabular}{|c|c|c|c|c|c|c|}
\hline Year & $\begin{array}{c}P(t) / \\
10^{8}\end{array}$ & $\begin{array}{c}A(t) / \\
10^{13} \\
\text { Yuan } \\
\end{array}$ & $\begin{array}{c}\mathcal{E} f \operatorname{con}(t) \\
/ \%\end{array}$ & $\begin{array}{c}\text { Eecon }(t) \\
/ \%\end{array}$ & $\begin{array}{c}\lambda_{e}(t) / \\
10^{8} \\
\text { Yuan }\end{array}$ & $\begin{array}{c}\lambda_{\text {total }}(t) / \\
10^{8} \\
\text { Yuan }\end{array}$ \\
\hline 03 & 12.923 & 1.37 & 17.5 & 15.6 & 3803.9 & 5508.4 \\
\hline 04 & 12.999 & 1.62 & 16.8 & 15.4 & 5064.2 & 7504.8 \\
\hline 05 & 13.076 & 1.87 & 13.5 & 13.5 & 6777.8 & 10205.6 \\
\hline 06 & 13.145 & 2.19 & 9.6 & 14.6 & 7605.8 & 11826.3 \\
\hline 07 & 13.213 & 2.70 & 8.7 & 14.4 & 8253.2 & 13698.6 \\
\hline 08 & 13.280 & 3.20 & 2.9 & 5.6 & 9443.7 & 16345.5 \\
\hline 09 & 13.345 & 3.49 & 4.8 & 7.2 & 11790 & 19477.9 \\
\hline 10 & 13.409 & 4.13 & 7.3 & 13.2 & 12879 & 21627.1 \\
\hline 11 & 13.474 & 4.89 & 7.3 & 12.1 & 12848 & 23045.6 \\
\hline 12 & 13.540 & 5.40 & 3.9 & 5.9 & 14553 & 25499.8 \\
\hline 13 & 13.607 & 5.95 & 3.7 & 8.9 & 16937 & 29008.9 \\
\hline 14 & 13.678 & 6.44 & 2.1 & 4.0 & 19674 & 31514.9 \\
\hline 15 & 13.746 & 6.89 & 1.0 & 2.9 & 22592 & 32562.1 \\
\hline 16 & 13.827 & 7.44 & 1.4 & 5.0 & 24773 & 32837.4 \\
\hline 17 & 13.905 & 7.95 & 1.5 & 5.6 & 26343 & 34654.6 \\
\hline
\end{tabular}
improved BP neural network.

TABLE I. DATA ELECTRICAL ENERGY SUBSTITUTION FROM

Note: The above data are from the China Statistical Yearbook 2003-2017.

The accumulated electric energy substitution amount over the years is obtained by summing the direct substitution 
quantity and the indirect substitution quantity. The result is shown in Fig. 5.

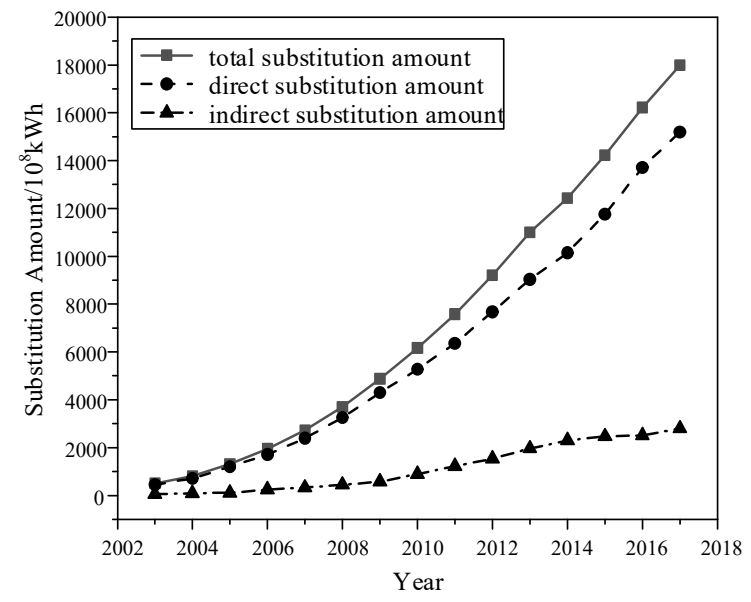

Fig. 5. Annual amount of electrical energy substitution.

The quantitative values of population, wealth, and technological development were estimated by Logistic curve fitting [17]. Policy support was estimated by its polymorphic function because of its developmental volatility [18]. To prevent over-fitting, choose quadratic function to estimate. The fit curve is shown in Fig. 6.

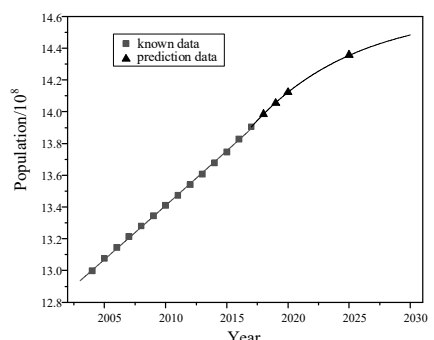

(a)

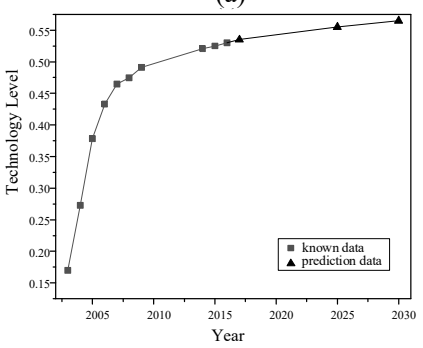

(c)

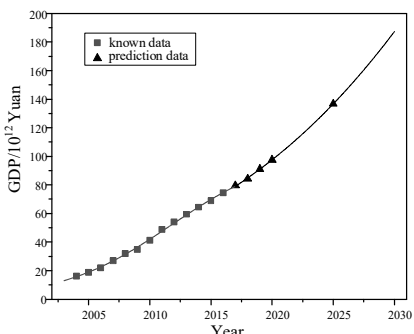

(b)

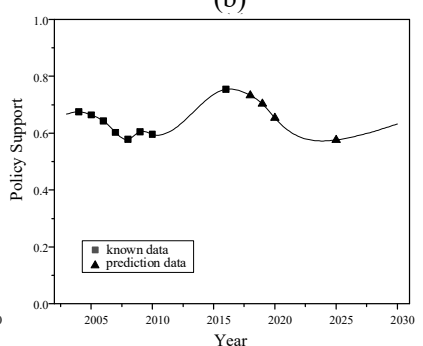

(d)
Fig. 6. Diagram of fitting and estimation of the influencing factor of Electrical energy substitution: a) population; b) wealth; c) technology; d) policy support.

The designed BP network has 1 input layer, 2 hidden layer $\mathrm{s}$ and 1 output layer. The number of nodes in the input layer is 4 , the number of nodes in the first layer of the hidden layer is 20 , the number of nodes in the second layer of the hidden layer is 40 , and the number of nodes in the output layer is 2 , which are direct substitution amount and indirect substitution amount. The upper limit of training times is set to 50,000 times, the training precision is $10^{-5}$, the weight coefficient correction constant is 0.8 , the initial learning rate is $0.1,70 \%$ of the known data is randomly assigned to the learning data, and $30 \%$ is the test data for BP neural network training. After 15205 iterations of training, the system accuracy reaches 10-5, and the system error is shown in Table II.
TABLE II. THE RELATIVE ERROR OF IMPROVED BP NETWORK

\begin{tabular}{|c|c|c|c|c|}
\hline Year & $\begin{array}{c}\Delta \boldsymbol{E}_{\boldsymbol{d}}(\boldsymbol{t}) / \\
\mathbf{1 0}^{\mathbf{8}} \mathbf{k W h}\end{array}$ & $\begin{array}{c}\text { Relative } \\
\text { error } \\
\mathbf{1 0}^{-4}\end{array}$ & $\begin{array}{c}\boldsymbol{\Delta} \boldsymbol{E}_{\text {in }}(\boldsymbol{t}) / \\
\mathbf{1 0}^{\mathbf{8}} \mathbf{k W h}\end{array}$ & $\begin{array}{c}\text { Relative } \\
\text { error/ } \\
\mathbf{1 0}^{-4}\end{array}$ \\
\hline 04 & 259.946 & 40.21 & 40.958 & 37.34 \\
\hline 05 & 490.923 & -1.57 & 19.181 & -1.76 \\
\hline 06 & 506.781 & 3.58 & 131.604 & 2.17 \\
\hline 07 & 686.502 & 4.35 & 80.418 & 2.75 \\
\hline 08 & 861.021 & -2.73 & 122.692 & 5.23 \\
\hline 09 & 1043.938 & 1.67 & 129.632 & 1.87 \\
\hline 10 & 978.28 & 1.65 & 311.984 & 2.14 \\
\hline 11 & 1078.692 & 0.66 & 333.505 & 0.64 \\
\hline 12 & 1316.831 & 0.43 & 313.685 & 0.97 \\
\hline 13 & 1357.318 & 1.20 & 433.389 & 1.32 \\
\hline 14 & 1111.028 & -0.89 & 331.437 & -0.73 \\
\hline 15 & 1618.44 & 0.40 & 173.211 & 0.65 \\
\hline 16 & 1852.557 & 0.44 & 137.052 & 0.47 \\
\hline 17 & 1579.132 & 0.60 & 189.346 & 0.32 \\
\hline
\end{tabular}

It can be seen that except for the error of the output data in 2004 reaching the order of $10^{-3}$, the rest of the data is in the order of $10^{-4} \sim 10^{-5}$, and the error precision is calculated by the square term, so the precision reaches $10^{-5}$.

The average annual prediction growth is used as the prediction result evaluation index:

$$
\begin{gathered}
\sigma=\frac{\sum_{j=1}^{2} \sum_{t=2018}^{2030}\left|\frac{y_{j}^{t}}{E_{s u b}(t-1)+y_{j}^{t}}\right|}{13} \times 100 \%, \\
E_{s u b}(t)=E_{s u b}(t-1)+y_{j}^{t},
\end{gathered}
$$

where $t \geq 2018$. It will be a reasonable prediction when $\sigma$ is at $3 \%-6.5 \%[10]$.

In the improved BP algorithm used in this paper, the adaptive learning rate is introduced, and the learning rate is continuously adjusted with the number of iterations. Table III compares the improved BP algorithm with the non-improved BP algorithm.

TABLE III. COMPARISON BETWEEN IMPROVED BP ALGORITHM

\begin{tabular}{|c|c|c|c|}
\multicolumn{4}{c}{ AND ORIGINAL BP ALGORITHM. } \\
\hline Method & $\boldsymbol{\eta}$ & Number of iterations & $\sigma$ \\
\hline Improved BP & $/$ & 15205 & $4.35 \%$ \\
\hline \multirow{3}{*}{ BP } & 0.01 & 45965 & $3.28 \%$ \\
\cline { 2 - 4 } & 0.05 & 23119 & $4.25 \%$ \\
\cline { 2 - 4 } & 0.1 & 8781 & $0.11 \%$ \\
\hline
\end{tabular}

It can be seen from the table that the number of iterations of the improved BP algorithm is relatively small, and the prediction result is reasonable. The non-improved algorithm for determining the learning rate is reasonable when the learning rate is 0.01 and 0.05 . When the learning rate is 0.1 , the over-predicted prediction distortion occurs. The comparison shows that the improved BP algorithm can reduce the number of iterations and achieve accurate prediction.

Figure 7 shows the learning rate change when the number of iterations increases at initial learning rates of $0.01,0.05$, and 0.1 respectively. It can be seen that the final learning rate 
tends to be between 0.03 and 0.06 .

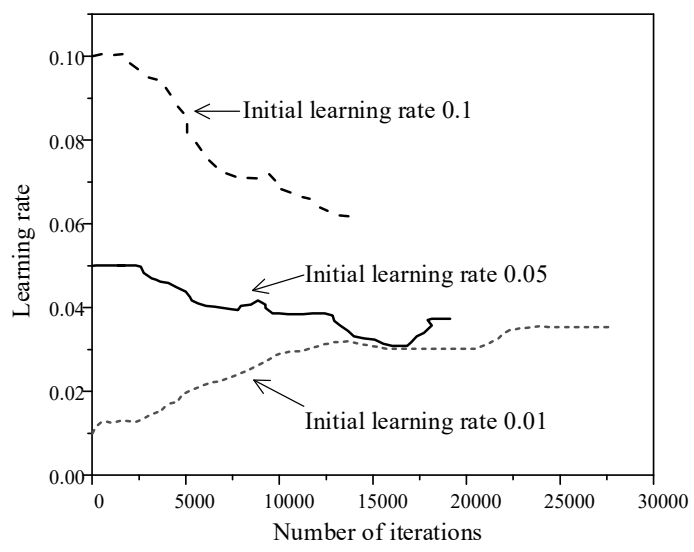

Fig. 7. Diagram of learning rate change under different initial values.

In this paper, when setting the BP output layer node, the two-node output is selected, which is the direct substitution incremental output and the indirect substitution incremental output respectively, and the two results are added to obtain the annual electric energy substitution amount increment. The reason is that the four variables of the input have different effects on the direct substitution increment and the indirect substitution increment, and the separate output is objective and reasonable. The table below gives a comparison of the two-node output versus the single-node output.

TABLE IV. COMPARISON OF TWO-NODE OUTPUT AND SINGLE-NODE OUTPUT.

\begin{tabular}{|c|c|c|c|}
\hline Output layer & $\sigma_{1}$ & $\sigma_{2}$ & $\sigma$ \\
\hline Two-node & $4.76 \%$ & $3.94 \%$ & $4.35 \%$ \\
\hline Single-node & $/$ & $/$ & $2.82 \%$ \\
\hline \multicolumn{2}{|l|}{ Note: $\sigma_{l}$ is average annual prediction growth of direct substitution, $\sigma_{2}$ is average annual }
\end{tabular}
prediction growth of indirect substitution.

It is known from Table IV that the average annual growth rate of single-node output is below $3 \%$, and the prediction amount is low. Therefore, the two-node output is selected in this paper.

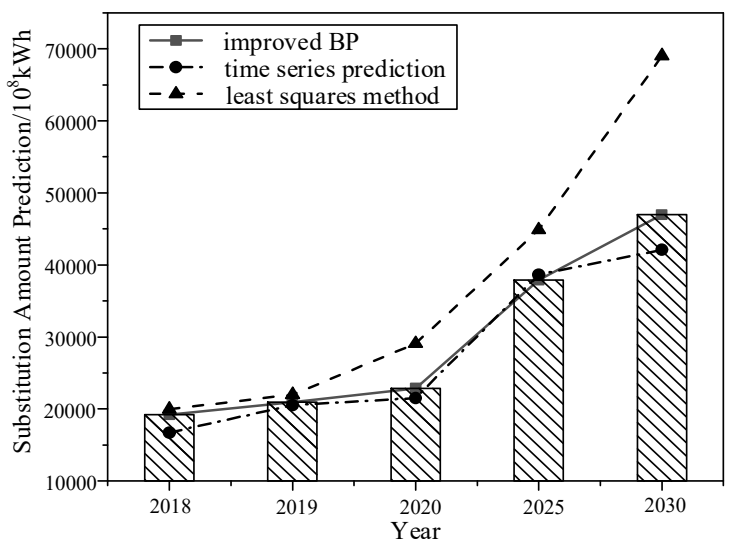

Fig. 8. Comparison of the three methods of Electrical energy substitution potential prediction.

The improved BP neural network algorithm prediction results are compared with the time series prediction algorithm [19] and the least squares method [20], as shown in Fig. 8. It can be seen that the time series prediction algorithm has greater volatility, the least squares method increases too much, and the predicted annual growth rate reaches $7.9 \%$. The result of improving the BP network algorithm is the most reasonable.

The improved BP neural network algorithm predicts the future energy substitution amount as shown in Fig. 8. It is predicted that the substitution amount of electric energy from 2018 to 2020 is 1922.1764 billion kWh, 2092.1865 billion $\mathrm{kWh}$, and 2287.6237 billion $\mathrm{kWh}$, the substitution amount of electric energy in 2025 is 3787.6654 billion $\mathrm{kWh}$, and the substitution amount of electric energy in 2030 is 4696.8892 billion $\mathrm{kWh}$.

\section{CONCLUSIONS}

This paper studies the issue of electrical energy substitution and its potential prediction. Firstly, the electric energy substitution is classified in different styles, and the electric energy substitution quantity model is established to quantify the substitution potential. Secondly, the EPATR model and the various influencing factors are established. Then, the main factors are estimated by the Logistic curve, and the BP neural network is established and improved. Additional momentum factors and adaptive learning rate are introduced and relevant parameters are set. Then the improved BP learns and trains with known data, and reaches the training accuracy, predicts the substitution amount of electric energy in the next decade. The example shows that the improved BP algorithm has higher computational accuracy, fewer iterations, and reasonable prediction results. The prediction results are compared with time series prediction and least squares. The result proves that the prediction result of this method is accurate and has guiding significance for the "fourteenth five-year" electric energy substitution work in China.

\section{REFERENCES}

[1] H. Yin, "Research on the method of energy replacement in other energy sources under the environment of energy saving and emission reduction", M.S. thesis, Dept. Electron. Eng., North China Electric Power Univ., Beijing, China, 2013

[2] Y. Gao, "Research on electric energy substitution mode and adaptation method under the background of abandoned wind and abandoned light”, M.S. thesis, Dept. Electron. Eng., North China Electric Power Univ., Beijing, China, 2017.

[3] Y. Li, Z. Chen, "Research on regional electric energy substitution potential evaluation based on TOPSIS method of connection degree optimization", Power System Technology, vol. 1, pp. 1-10, 2018. DOI: 10.1155/2018/3834921.

[4] D. Cao, Y. Yuan, Z. Li, “Alternative application of electric energy and benefit evaluation", Power Grid and Clean Energy, vol. 27, pp. 30-34, 2011.

[5] Y. Shan, J. Zhao, D. Jia, "Analysis method of electric energy substitution potential based on STIRPAT-ridge regression", Supply and Electricity, vol. 1, pp. 68-73, 2018.

[6] Y. Sun, S. Zhou, Y. Shan, "Analysis of electric energy substitution potential under multiple scenarios”, Power System Technology, vol. 41, pp. 118-123, 2017.

[7] Y. Sun, M. Shi, Y. Shan, "Analysis method of electric energy substitution potential based on particle swarm optimization support vector machine", Power System Technology, vol. 41, pp. 1767-1771, 2017.

[8] H. Xia, H. Lin, W. Zhang, "Research on regional electric energy substitution development potential based on multi-model", Science and Technology Management Research, vol. 4, pp. 241-246, 2018.

[9] L. Cheng, W. Jin, M. Zhong, "Research and design of electric energy replacement standard system”, Smart Grid, vol. 4, pp. 875-880, 2016.

[10] X. Liang, W. Lu, H. Zhou, "Electric energy substitution in energy transformation", Smart Grid, vol. 3, pp. 1192-1196, 2015.

[11] Y. Sun, P. Xu, Y. Shan, 'Internet +' electric energy alternative 
development route under the background of electricity sales reform", Power System Technology, vol. 40, pp. 3648-3654, 2016.

[12] Y. Shen, "Research on regional energy efficiency decomposition in China based on IPAT-LMDI model", M.S. thesis, Dept. Electron. Eng., North China Electric Power Univ., Beijing, China, 2017.

[13] L. Meng, C. Li, G. Hu, "Research on population structure prediction in China based on PDE model", China Population, Resources and Environment, vol. 24, pp. 132-141, 2014.

[14] R. Zhang, Z. Xu, G. Huang, "Global convergence of online BP training with dynamic learning rate", IEEE Trans. Neural Networks and Learning Systems, vol. 23, no. 2, pp. 330-341, 2012. DOI: 10.1109/TNNLS.2011.2178315.

[15] Y. Noorollahi, M. A. Jokar, A. Kalhor, "Using artificial neural networks for temporal and spatial wind speed forecasting in Iran", Energy Conversion and Management, vol. 115, pp. 17-25, 2016. DOI: 10.1016/j.enconman.2016.02.041.

[16] F. Yang, H. Cho, H. Zhang, "Artificial neural network (ANN) based prediction and optimization of an organic rankine cycle (ORC) for diesel engine waste heat recovery", Energy Conversion and Management, vol. 164, pp. 15-26, 2018. DOI: 10.1016/j.enconman.2018.02.062.

[17] P. Peduzzi, J. Concato, E. Kemper, "A simulation study of the number of events per variable in logistic regression analysis", Journal of Clinical Epidemiology, vol. 49, no. 12, pp. 1373-1379, 1996. DOI: 10.1016/S0895-4356(96)00236-3.

[18] A. J .C .Varandas, J. N. Murrell, "Choosing points in potential energy surfaces for fitting polynomial functions: application of permutational symmetry", Chemical Physics Letters, vol. 84, no. 3, pp. 440-445, 1981. DOI: 10.1016/0009-2614(81)80381-8.

[19] W. Shi, K. Wu, D. Wang, "Short-term load forecasting of power system based on time series analysis and Kalman filter algorithm", Automation Technology and Application, vol. 37, pp. 9-12, 2018.

[20] Y. Zhang, T. Fearn, "A linearization method for partial least squares regression prediction uncertainty", Chemometrics and Intelligent Laboratory Systems, vol. 140, pp. 133-140, 2015. DOI: 10.1016/j.chemolab.2014.11.011. 